\title{
Effect of size and shape on the elastic modulus of metal nanowires
}

\author{
Lilian Maria Vogl ${ }^{1} \cdot$ Peter Schweizer ${ }^{1} \cdot$ Gunther Richter ${ }^{2} \cdot$ Erdmann Spiecker $^{1}$ (i)
}

Received: 6 May 2021 / Accepted: 1 July 2021 / Published online: 2 August 2021

(c) The Author(s) 2021

\begin{abstract}
Size effects decisively influence the properties of materials at small length scales. In the context of mechanical properties, the trend of 'smaller is stronger' has been well established. This statement refers to an almost universal trend of increased strength with decreasing size. A strong influence of size on the elastic properties has also been widely reported, albeit without a clear trend. However, the influence of nanostructure shape on the mechanical properties has been critically neglected. Here, we demonstrate a profound influence of shape and size on the elastic properties of materials on the example of gold nanowires. The elastic properties are determined using in-situ mechanical testing in scanning and transmission electron microscopy by means of resonance excitation and uniaxial tension. The combination of bending and tensile load types allows for an independent and correlative calculation of the Young's modulus. We find both cases of softening as well as stiffening, depending critically on the interplay between size and shape of the wires.
\end{abstract}

\section{Introduction}

In recent years, different techniques have been established for in-situ mechanical testing of nanostructures, revealing a size dependency of the properties commonly referred to as size effect. For plastic deformation, a general trend of "smaller is stronger" is well established [1-4]. Moreover, recent work [5] suggests, that shape may indeed play a role in determining the mode of plastic deformation. However, it is not yet clear if elasticity on small scales is influenced in a similar way as plasticity. While some researchers found a stiffening [6] with decreasing size, other groups report a softening effect [7]. Moreover, the shape has not been considered so far. Therefore, we present a correlative nanomechanical approach to evaluate the shape and size dependent elastic behavior of metal nanowires. Next to tensile testing $[8,9]$, bending $[10,11]$, compression [12], and nanoindentation [13], the measurement of resonance frequencies is an elegant and widely used method to determine the Young`s

Erdmann Spiecker

Erdmann.Spiecker@fau.de

1 Institute of Micro- and Nanostructure Research (IMN) and Center for Nanoanalysis and Electron Microscopy (CENEM), Interdisciplinary Center for Nanostructured Films (IZNF), Friedrich-Alexander-Universität ErlangenNürnberg, Cauerstrasse 3, 91058 Erlangen, Germany

2 Max Planck Institute for Intelligent Systems, Heisenbergstrasse 3, 70589 Stuttgart, Germany modulus of one dimensional nanostructures [14-16]. Critically, the accuracy of the data obtained from small-scale mechanical testing is strongly affected by several factors, such as the uncertainty of the geometric dimensions, especially the cross-sectional geometry of the nanowires [17], load misalignment [18], wire clamping [19], and focused ion beam (FIB) preparation artifacts [20]. All of these factors can lead to considerable error in the Young's modulus determination [21]. To further determine potentially small influences of size and shape on this property, it is critical, to minimize all sources of error. In this work, we, therefore, focused on resonance measurements, which exclude many of the aforementioned problems. Moreover, resonance is a non-destructive method, enabling the cross-correlation of results with subsequent tensile testing on the same wire.

\section{Materials and methods}

\section{Sample system}

We analyzed the Young's modulus of freestanding gold nanowires grown by physical vapor deposition [22]. Gold was chosen as a model material due to its inertness to oxidation and well-known properties. The nanowires have a characteristic [110] growth direction. The samples exhibit a broad range of different sizes and shapes, which offers the opportunity to study and compare the influencing factors on 
the mechanical properties in detail (see Fig. S1). Moreover, some nanowires exhibit twin grain boundaries along the wire axis.

\section{Sample preparation}

Mechanical tests require isolated freestanding nanowires firmly clamped on one side (see Fig. S2), and thus, a controlled sample preparation method is needed. Here, we introduce a workflow based on a controlled wire picking routine in the SEM (see Figs. 1a-c, S3), which does not require the use of a FIB and which enables the subsequent testing of the elastic mechanical properties with resonance measurements and tensile testing. A tungsten tip mounted on a micromanipulator, is aligned parallel to the desired nanowire and brought into mechanical contact. After the alignment of the tip, the nanowire is clamped with localized electron beaminduced carbon deposition. The carbon deposition must be carefully done, to ensure an appropriate clamping width with good connection of the nanowire end to the tip. For each wire, we chose the optimum clamping parameter according to Qin et al. [19]. We analyzed the frequency shift induced by the clamping to make sure that the parameters are optimal (see Figure S4). After deposition, the nanowire is gently pulled off the substrate usually resulting in a localized plastic event close to or at the substrate. After picking, the size of the nanowire can be assessed (see Fig. 1c) to estimate the range of expected resonance frequencies and correlate the shape to the vibration directions in dependence of the nanowire dimensions.

\section{Resonance measurements}

For the resonance measurements, a second manipulator tip is arranged perpendicular to the first one, enabling electrical excitation [23]. In Fig. 1d, a schematic of the experimental setup in the SEM with the applied sinusoidal potential generated by a waveform generator is shown. The electric
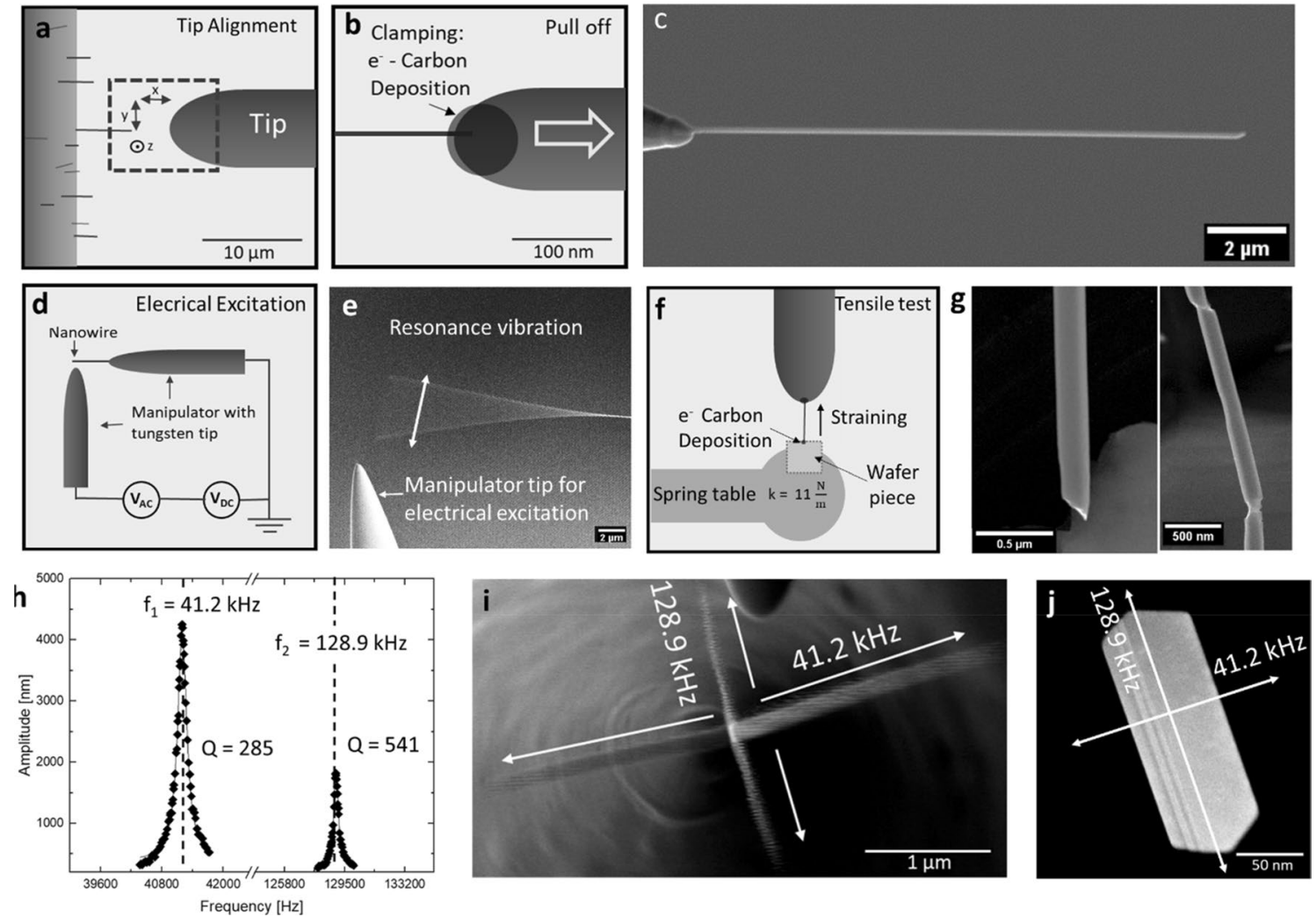

Fig. 1 a Sample preparation with $\mathrm{nm}$ precise alignment using a manipulator tip. b clamping of the nanowire with electron beaminduced carbon deposition. c controlled pulling off the nanowire from the substrate. Exemplary SEM image of a picked nanowire with a sheared nanowire end. d experimental setup in the SEM, using electrical excitation for the resonance measurements. e exemplary SEM image of a nanowire in resonance frequency showing an interference pattern between the scanning electron beam and the resonance vibration of the nanowire. $\mathbf{f}$ subsequent tensile testing of the nanowire with a calibrated spring table. $g$ SEM images of the nanowires after the destructive tensile test, with plastic deformation. h Amplitude-frequency plot of an Au nanowire with two resonance vibrations. In $\mathbf{i}$, the corresponding vibrations are shown in top view (overlaid SEM image of both resonance vibrations). The resonance vibrations $\mathrm{f} 1$ and $\mathrm{f} 2$ are assigned to the corresponding nanowire dimensions. $\mathbf{j}$ STEM image of the same nanowire in cross section, showing the microstructure 
potential results in a periodic attractive force between tip and nanowire [24] which leads to an induction of vibrations. When a frequency is applied to the nanowire, which matches one of its resonance frequencies, the vibration amplitude of the nanowire is greatly increased. This change in amplitude is readily visible in SEM as shown in Fig. 1e. By sweeping through a frequency range around the resonance frequency and measuring the amplitude of the wire vibration, frequency-amplitude diagrams are obtained (see Fig. 1h and videos V1 and V2). Care has to be taken to assign the correct vibrational mode to each peak. Considering the cross-sectional geometry, there are two (neglecting twist and breathing modes) independent resonance frequencies for rectangularly shaped nanowires. Due to the electrical excitation, additional peaks appear at exactly half of the resonance frequencies, which can be explained by the applied force on a nanowire during excitation [25, 24] (see Figure S5). Furthermore, next to the fundamental 1st mode of vibration, also higher modes in resonance can be reached, showing the characteristic vibrational nodes (see Figure S6). For the direct visual assignment of the vibration directions to the specific nanowire shape, the sample can be tilted, which enables us to observe the nanowire from above. Figure 1i shows the two fundamental resonance frequencies of an exemplary wire superimposed on each other. They are excited at different frequencies of $41.2 \mathrm{kHz}$ and $128.9 \mathrm{kHz}$ and are orthogonal to each other (see Figure S7 and video V3). Figure $1 \mathrm{j}$ shows a STEM image of the cross section of the same nanowire taken after testing. With the direct information of the characteristic vibration directions, shown in Fig. 1i, the resonance frequencies can be unequivocally assigned to the corresponding nanowire dimensions. The STEM image reveals several twin grain boundaries along the wide side of the nanowire, which might be one of the reasons for the difference in quality factor for the observed different vibrational modes. The additional grain boundaries act as barriers for the vibration motion across the wide side of the nanowire and the resonance amplitude at $41.2 \mathrm{kHz}$ gets damped. The second vibration direction at $128.9 \mathrm{kHz}$ has double the quality factor, indicating that it is not affected by the twin structure.

\section{Tensile testing}

Taking advantage of the non-destructive resonance measurements, the workflow can be extended to subsequent tensile testing of the same nanowire (see Fig. 1f). After completion of the resonance measurement, the nanowire on the micromanipulator tip is carefully attached to a calibrated spring table (spring constant $k=11 \mathrm{~N} / \mathrm{m}$ ) and then strained along the axis in-situ in SEM (see exemplarily Fig. 3c for the setup). By moving the manipulator, the nanowire is strained (strain rate $2-3 \times 10^{-4} \mathrm{~s}^{-1}$ ) and plastically deformed until the wire tears apart (see video V4). The SEM images in Fig. 1g show representative deformed nanowires after the straining process. Both force and displacement are obtained by template-based, sub-pixel accurate digital image correlation. The force is calculated with the spring constant of the spring table and the displacement of the sample. For the strain, both ends of the wires are tracked individually to rule out any influence by compliance effects of the test setup. With this, the effective elongation and, therefore, the strain on the nanowire can be accurately measured. After the tensile test, a cross section for further TEM investigations is prepared by FIB lift-out of an undeformed nanowire piece. With the exact wire geometry, the force-displacement curve is translated into a stress-strain curve. By using the linear part of the stress-strain curves, the elastic modulus can be evaluated and related to size and shape.

\section{Instruments}

The in-situ resonance and tensile tests are performed in a FEI Helios Nanolab 660 using Kleindiek MM3A-EM micromanipulators and a Kleindiek spring table with a spring constant of $11 \mathrm{~N} / \mathrm{m}$. The afterwards prepared nanowire cross sections are analyzed in a FEI Titan Themis ${ }^{3} 300$ transmission electron microscope. For the resonance measurements, an arbitrary waveform generator Rigol DG1022Z with a frequency range of $1 \mu \mathrm{Hz}$ to $25 \mathrm{MHz}$ and a sample rate of $200 \mathrm{MSa} / \mathrm{s}$ is used. A sinusoidal wave with $2 \mathrm{Vpp}$ is applied for excitation, and the frequency is swept through the interesting frequency range $(1 \mu \mathrm{Hz}$ resolution, range normally between 1 and $200 \mathrm{kHz}$ ). To set the offset level/ the static voltage $V_{s}$, a Keithley sourcemeter 2400 is used (normally between 0-2 V). For modeling the nanowire, the software Abaqus/CAE 2017 from the Dassault Systèmes Simulia Group is used.

\section{Results}

\section{Resonance measurements}

The workflow of picking and measuring the resonance frequencies followed by TEM investigation of the cross section has been performed for many wires of different shapes and sizes. On basis of the presented accurate characterization and assignment of the vibrational modes, the elastic properties of the nanowire in [110] direction can be calculated. We use classical beam theory according to Euler-Bernoulli for the calculation of the Young's modulus, which assumes a long and slender beam with relative small deformations [26]. With nanowire lengths in the range of $10 \mu \mathrm{m}$ up to $25 \mu \mathrm{m}$, thicknesses below $1 \mu \mathrm{m}$ and strains during vibration well below $0.1 \%$ (see Fig. S8), the boundary condition for 
the application of the theory is well fulfilled. The Young's modulus $\mathrm{E}$ can then be calculated though the equation:

$E=\frac{4 \pi^{2} \cdot f^{2} \cdot L^{4} \cdot A \cdot \rho}{\beta^{4} \cdot I}$

with the resonance frequency $f$, wire length $L$, crosssectional area $A$, density $\rho$, second moment of area $I$ and the factor $\beta=1.875$ for the fundamental first resonance mode. From the equation, it is immediately apparent that the wire dimensions need to be precisely determined to get an accurate measure of the Young's modulus. Especially the cross-sectional area and the second moment of area can only be accurately determined by the direct measurement of the cross section of the nanowires with sub-nm resolution. This is done by preparing a thin lamella from each tested nanowire and taking high-resolution STEM images (exemplarily shown in Fig. 1j). We have to emphasize here that any rough ball parking of the wire dimensions leads to critical errors in the measured elastic modulus. Based on the TEM analysis, we can achieve a maximum uncertainty of around $16 \%$ (see
Fig. S9), making this step crucial in our study. To characterize the vibration direction of the nanowires, Abaqus' FEM analyses were performed, where the wire is modeled based on the microscopic data (see Fig. S10).

Figure 2 presents an overview of exemplary nanowires grouped by their size and shape based on the TEM analysis of the cross-sections. Since each wire has two orthogonal vibration directions, two independent measures of $E$ are obtained from the same wire. Conventionally, it would be expected that both measures should be identical as both are approximately only dependent on the stiffness along the wire axis. However, we find this to be true only for wires with a cross-sectional aspect ratio close to 1 . For wires with a rectangular cross section, two different stiffness values are obtained, one for each vibrational direction (see Figure $\mathrm{S} 11$ ). This splitting can be attributed to different characteristic sizes in the different directions. We therefore classified the measured stiffness values according to the size of the wire cross section in vibration direction, to work out a size dependency. In the columns in Fig. 2, the area of the nanowire cross-section increases from top to bottom. Following

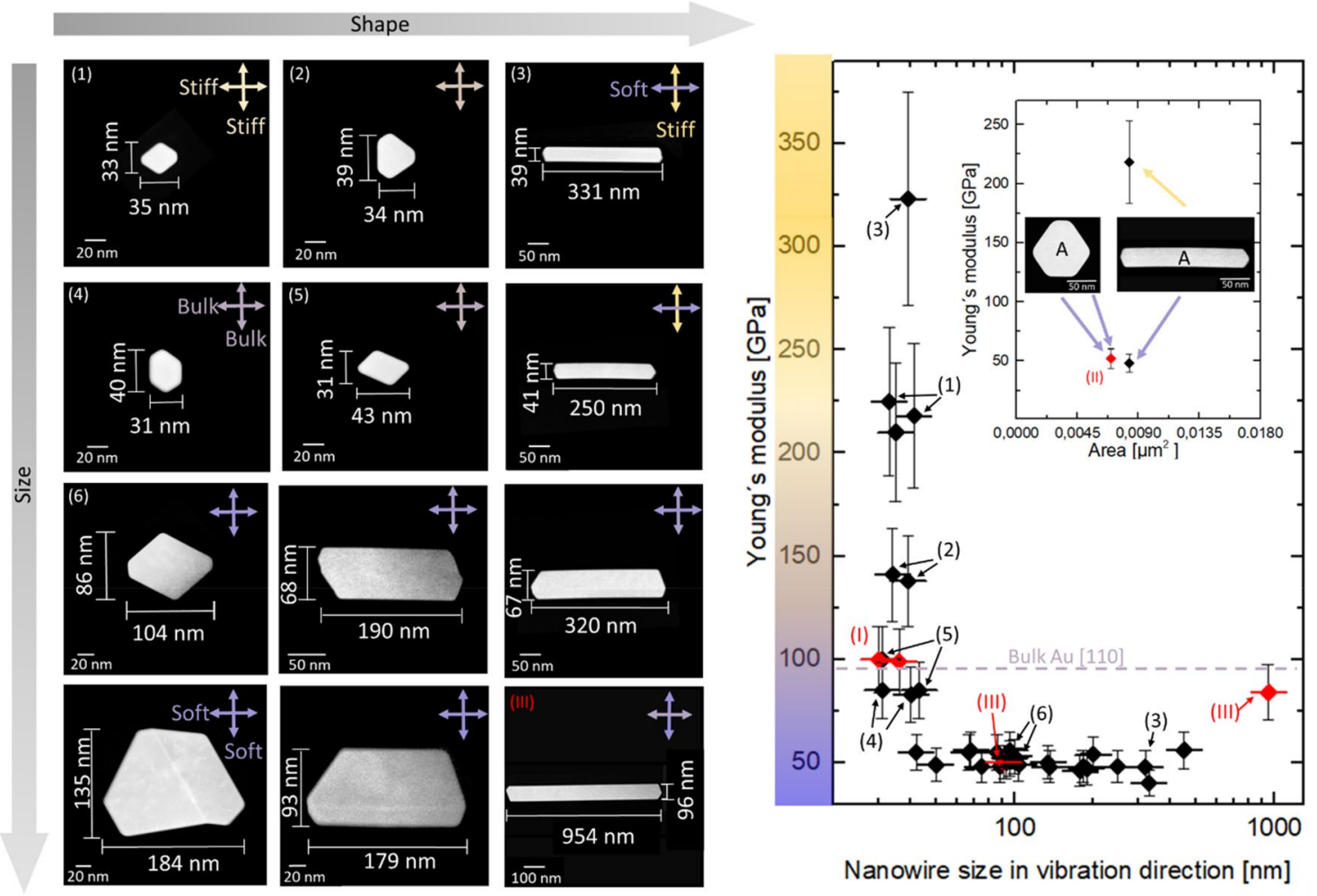

Fig. 2 The nanowires are grouped according to their cross-sectional size and shape: The relative size of the nanowires increases in row direction, and the shape of the nanowires is grouped from left (regular shape) to right (high-aspect ratio rectangular shape). The arrow cross in the upper right marks the vibration directions, and the color code is defined by the characteristic Young's modulus in the graph on the right side, where the Young's modulus is plotted vs. the rela- tive nanowire size (see also Supporting Information S12). The numbered images serve to highlight specific examples. The inset shows the shape dependency of the Young's modulus of two nanowires with nearly the same area. The sizes of the nanowires are measured as the greatest possible distance in vibration direction. The red values (roman numerals) are also tested in tensile loading 
the rows from left to right, the shape of the nanowires changes to flat, belt-like structures. Some nanowires show twin grain boundaries along the wire axis. In the following description, we do not differentiate between wires with and without twins, as we do not see a significant difference in the Young's modulus. Based on the resonance frequencies, the Young's moduli of the wires in the two directions are calculated, and their respective magnitude is indicated by the colored arrow cross in each image. The arrows indicate the vibration direction, and the color scale is defined in the $y$-axis of the graph on the right, as shown in Fig. 2. The graph shows all the measured Young's moduli versus the characteristic size in vibration direction. Starting with wires of equal aspect ratio, coming from the bulk side a decrease in the Young's modulus is found (see exemplary NW (6)). Decreasing the size of the nanowires further (below $40 \mathrm{~nm}$ ), the Young's modulus increases again to the range of the bulk value [see exemplary NW (4)] and then further to values of $140 \mathrm{GPa}$ [see exemplary NW (2)] and even $220 \mathrm{GPa}$ [see exemplary NW (1)]. For these wires, both resonance directions result in a similar value for the Young's modulus. For wires with increasing aspect ratio, the shape gets more and more important. In the inset of the graph in Fig. 2, two nanowires with nearly the same size in terms of their crosssectional area are compared. By changing the regular shape of the nanowire to a flat rectangular cross section, a splitting of the Young's modulus for different load directions is observed. This means that the mechanical properties of such nanostructures cannot be simply related to a single characteristic size. Instead the shape has to be critically taken into account. This trend is seen for all belt-like nanowires, with the individual stiffness values following the general size relationship. For instance, NW (III) shows a bulk-like stiffness of around $80 \mathrm{GPa}$ along its long direction which extends to a length of just close to $1 \mu \mathrm{m}$. In the orthogonal, much shorter direction $(96 \mathrm{~nm})$, a softening down to around $50 \mathrm{GPa}$ is observed. For much smaller belts, such as in the case of NW (3), the long side shows a softening down to $50 \mathrm{GPa}$, with the short side becoming much stiffer reaching values of over $300 \mathrm{GPa}$.

\section{Subsequent tensile testing}

With the strong discrepancies in stiffness in different bending directions, it was of further interest to characterize this property in a different load setting to cross-correlate the results. Tensile loading only results in a single value for the stiffness along the wire direction, which should correlate in some way to the values found for different bending directions. In Fig. 3, three representative nanowires with different elastic behaviors are shown. According to the characteristic resonance frequencies, wire $\mathrm{I}$, with dimensions in the range of $30-36 \mathrm{~nm}\left(\right.$ Area $\left._{\mathrm{Wirel}}=947 \mathrm{~nm}^{2}\right)$, has a stiffness in the range of $100 \mathrm{GPa}$ in both bending directions. The subsequent tensile test results in the same value, which confirm not only the validity of the resonance measurements but also solidifies the size-stiffness relationship. Increasing the nanowire size to dimensions in the range of 96-100 nm $\left(\right.$ Area $_{\text {WireII }}=6989 \mathrm{~nm}^{2}$ ) leads to a softening effect compared to the bulk value (see Fig. 3 Wire II). Both, the tensile test and the resonance analysis, result in an overall Young's modulus in the range of 52-55 GPa. Wire I and wire II exhibit a rather regular shape with cross-sectional aspect ratio close to 1 , enabling the clear assignment of one single Young's modulus to both of the measured resonance frequencies, which is further confirmed by the additional tensile tests of the same nanowires. In contrast to that, wire III has a belt-like structure with cross-sectional dimensions of

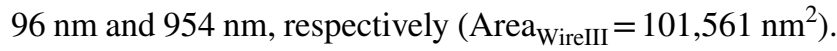
Reaching nearly $1 \mu \mathrm{m}$ in one direction, the stiffness is in the range of the bulk value of gold with $84 \mathrm{GPa}$, according to the corresponding resonance vibration at $226 \mathrm{kHz}$. Evaluating the resonance frequency of $20 \mathrm{kHz}$ in the $96 \mathrm{~nm}$ thin direction, we obtain a Young's modulus of $56 \mathrm{GPa}$, which is in the softening range. In tension, however, we obtain a value of $78 \mathrm{GPa}$, which lies in between the two values obtained from resonance. For this wire, it is clearly not possible to relate the size effect of stiffness to a single characteristic wire dimension, instead the shape has to be considered even in uniaxial tension.

\section{Discussion}

In contrast to previous studies, we could not confirm a general trend of softening [7, 27] or stiffening [6] with decreasing size and neither a constant bulk-like value [28, 29]. Instead, we find two different regimes for the Young's modulus with decreasing size: a softening followed by a stiffening regime. Over the past years, many researchers have worked on theoretical models to describe the size effects in the elastic behavior of nanowires. The sizedependent change in elasticity has been attributed to one or more of the following factors: Loss of atomic coordination [30], electron density redistribution [31], lattice contraction due to compressive strain [32], surface relaxation processes [33], and facet energy [34]. He and Lilley presented a theoretical approach analyzing the influence of surface stress on static bending [35] and in resonance vibration [36]. Both cases are also studied extensively by Yun and Parks [37]. Depending on the boundary conditions, both, a stiffening [38] or softening [39] of metal nanowires are predicted. Experimentally, a similar controversial trend has been observed. Petrova et al. have shown a softening of Au wires [7] while Li et al. have seen a stiffening [6] or even bulk-like behavior [28]. The different models can 
a

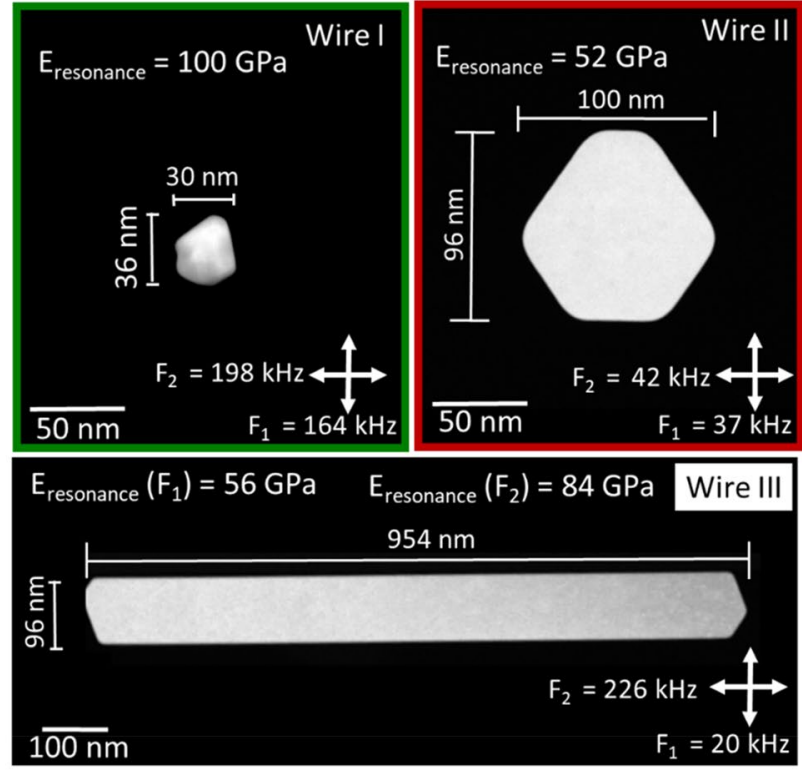

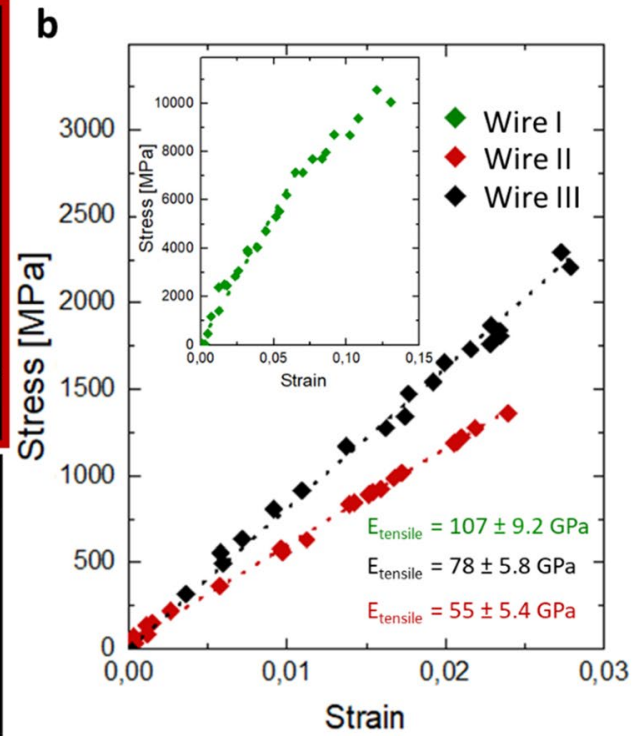
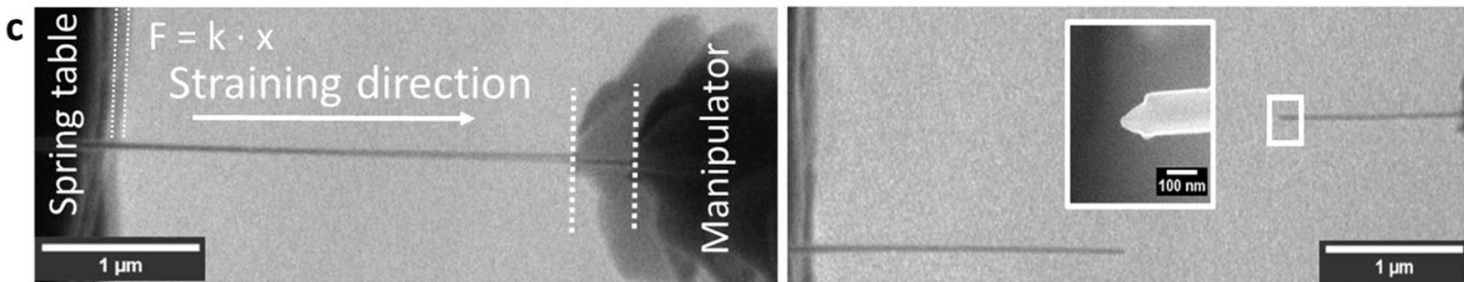

Fig. 3 a STEM images of nanowire cross sections of three different nanowires (Wire I-III). Wire 3 shows a splitting of the Young's modulus in resonance and in tensile testing a mixed value is obtained. b the Young's modulus of wire I (Stiffening) and Wire II (Softening) are verified in the corresponding stress-strain curves. c setup of the tensile test in SEM with calibrated spring table and manipulator. The wires show characteristic plastic deformation after the straining be mainly traced back to three fundamental theoretical concepts: Surface Stress Theory (SST) [40], Surface Elasticity Theory (SET) [41, 42], and Couple Stress Theory (CST) [43]. For fixed-free boundary condition, surface and residual stresses are released [44], whereas the theories of surface elasticity (SET) and couple stress (CST) are widely discussed to describe the behavior of nanobeams.

However, only a combination of multiple theories [44], in our case SET and CST, delivers a first model (taken from Abazari et al.) to describe our experimental data. Abazari et al. assume a rectangular beam with a thickness $\mathrm{h}$ and width $\mathrm{b}$. To simplify the mathematical description of the surface effects, they assume beams with a high aspect ratio with $b>>h$ and therefore only consider the thickness $h$ as critical parameter, see Fig. 4a. Based on a general core-shell approach, the surface and the core of the nanobeam are treated individually. The CST describes the influence of the core on the effective Young's modulus. The SET describes the mechanical properties of the surface. According to Abazari et al. the effective Young's modulus of the nanobeam is given as $E_{\text {eff }}=E_{\mathrm{Bulk}}-(\mathrm{SET})+(\mathrm{CST})$ :
$E_{\mathrm{eff}}=E_{\mathrm{Bulk}}-6 C_{\mathrm{s}}\left(\frac{1}{h}\right)+24 \frac{E}{1+v} l^{2}\left(\frac{1}{h}\right)^{2}$.

In this model, the surface is expected to have a reduced surface modulus $E_{\mathrm{s}}$, which is explained by the lower coordination number (softening effect). The surface elasticity $C_{\mathrm{s}}$ depends on the assumed layer thickness $\delta(\sim 1 \mathrm{~nm})$ and is defined as $C_{\mathrm{s}}=\delta \cdot\left(E_{\mathrm{Bulk}}-E_{\mathrm{s}}\right)$. On the other side, the core introduces a stiffening effect, which depends on the material-specific length scale parameter 1 [44]. The Poisson's ratio is described by $\nu$. The combination of both theories illustrates the competing interplay of the core and the surface and represents a first model including stiffening and softening mechanism at the same time (see Fig. $4 \mathrm{~b}$ and c). We extended the equation by Abazari et al. to include the increased impact of the surface elasticity caused by the width $b$ (see Supporting Information S12). In that term, we introduce an effective length $w=4 /\left(\frac{3}{h}+\frac{1}{b}\right)$, including the width $b$ and the beam thickness $h$. Figure $4 d$ shows the 
a
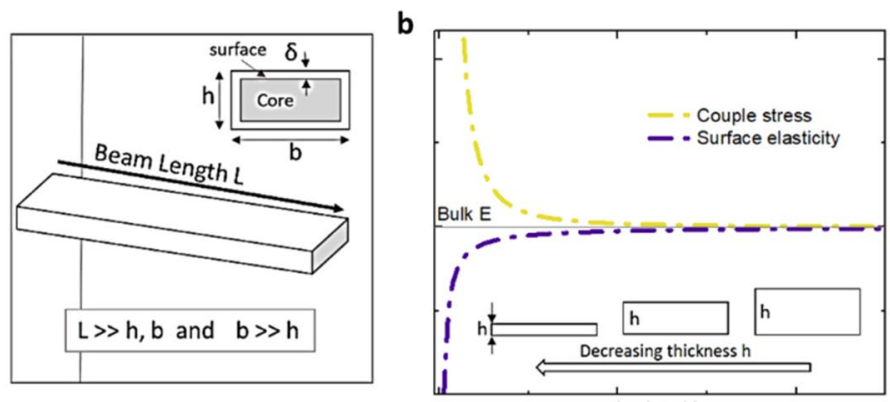

c

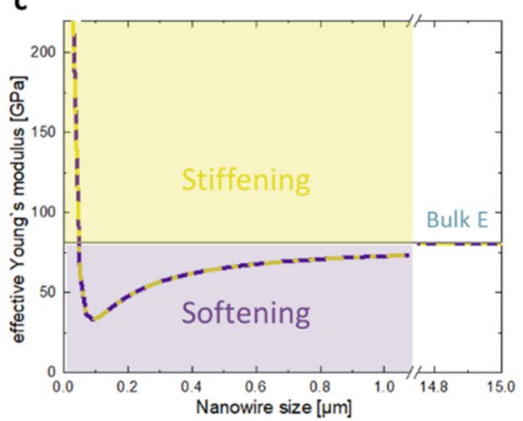

d

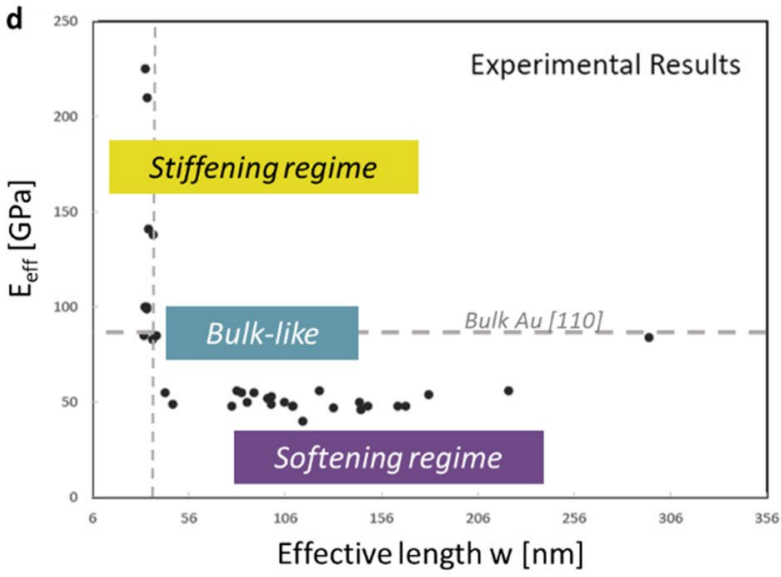

e

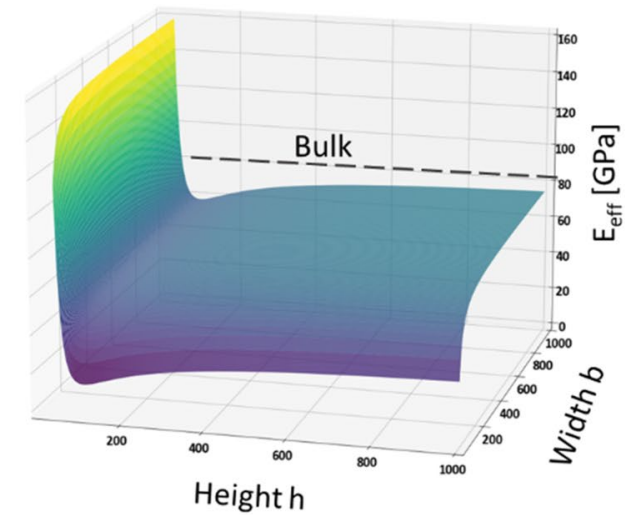

Fig. 4 Combination of Surface Elasticity (SET) and Couple Stress theory (CST) to describe the effective Young's modulus $E_{\text {eff }}$ of a rectangular beam in dependency of the width $b$ and the height $h$. a Schematic illustration of the assumed rectangular beam by Abazari et al. with $b>>h$ and $L>>h, b$. The beam is treated according to the general core-shell model, where the surface layer (thickness $\delta$ ) has different elastic properties compared to the bulk core. b Plot of the theoretical Young's modulus according to the coupled stress and the surface elasticity in dependence of the beam thickness $h$. A rectangu- lar beam with constant width $\mathrm{b}$ is considered. $\mathbf{c}$ Fitted experimental data with assumed rectangular cross section (fitting parameters $C_{\mathrm{S}}=1429 \frac{\mathrm{N}}{\mathrm{m}}, 1=16 \mathrm{~nm}$ ) according to the introduced combined model and equation by Abazari et al. d 2D representation using an introduced effective length $w=4 /\left(\frac{3}{h}+\frac{1}{b}\right)$. The experimental data show a softening and a stiffening regime. e 3D plot of the effective Young's modulus in dependence of the two parameters width $b$ and the height $h$ (fitting parameters $C_{\mathrm{S}}=700 \frac{\mathrm{N}}{\mathrm{m}}, 1=12 \mathrm{~nm}$ ) stiffening and softening regime of the effective Young's modulus in dependence of the effective length $w$ by assuming a rectangular beam. The 3D plot in Fig. 4e illustrates the effect of both geometric parameters $b$ and $h$ on the effective Young's modulus. Despite the rough assumption of rectangularly shaped beams, this first approach already supports the presented experimental data. Nevertheless, the exact shape of the nanowire has to be taken into account for a more complex description of the mechanical behavior including the shape effect. While we cannot determine exactly the involving surface effects, the provided experimental results show the size- and shapedependent interplay, which can lead to both softening and stiffening.

\section{Conclusion}

Using in-situ resonance measurements combined with subsequent tensile testing of nanowires in the SEM followed by a precise analysis of the shape and microstructure in TEM allowed us to characterize the elastic properties regarding size and shape. We find not only a size-dependent trend of softening and stiffening but also a clear influence of the shape. Wires with a rectangular cross section exhibit different Young's moduli in different bending directions. In tension, a mixture of both values is obtained. This study shows that not only size, but also shape is critically important for the elastic properties of 
nanostructures. We believe that this shape effect has to be considered in future modelling and experimental work.

Supplementary Information The online version contains supplementary material available at https://doi.org/10.1557/s43580-021-00103-3.

Acknowledgments The authors gratefully acknowledge funding by the DFG through projects GRK 1896 and SFB 953. The authors like to thank Ludwig Herrnböck for fruitful discussions. The authors declare no competing interests.

Author contributions LMV and PS contributed equally to the study. All authors discussed the results and commented on the manuscript.

Funding Open Access funding enabled and organized by Projekt DEAL.

\section{Declarations}

Conflict of interest The authors declare no competing interests.

Open Access This article is licensed under a Creative Commons Attribution 4.0 International License, which permits use, sharing, adaptation, distribution and reproduction in any medium or format, as long as you give appropriate credit to the original author(s) and the source, provide a link to the Creative Commons licence, and indicate if changes were made. The images or other third party material in this article are included in the article's Creative Commons licence, unless indicated otherwise in a credit line to the material. If material is not included in the article's Creative Commons licence and your intended use is not permitted by statutory regulation or exceeds the permitted use, you will need to obtain permission directly from the copyright holder. To view a copy of this licence, visit http://creativecommons.org/licenses/by/4.0/.

\section{References}

1. S.S. Brenner, Tensile strength of whiskers. J. Appl. Phys. 27(12), 1484-1491 (1956)

2. M.K. Kini, G. Dehm, C. Kirchlechner, Size dependent strength, slip transfer and slip compatibility in nanotwinned silver. Acta Mater. 184, 120-131 (2020)

3. R. Schwaiger, L.R. Meza, X. Li, The extreme mechanics of micro- and nanoarchitected materials. MRS Bull. 44(10), 758765 (2019)

4. D. Kiener, A.M. Minor, Source truncation and exhaustion: insights from quantitative in situ TEM tensile testing. Nano Lett. 11(9), 3816-3820 (2011)

5. S. Yin et al., Transition of deformation mechanisms in singlecrystalline metallic nanowires. ACS Nano 13(8), 9082-9090 (2019)

6. X. Li et al., Direct nanomechanical machining of gold nanowires using a nanoindenter and an atomic force microscope. J. Micromech. Microeng. 15(3), 551-556 (2004)

7. H. Petrova et al., Crystal structure dependence of the elastic constants of gold nanorods. J. Mater. Chem. 16(40), 3957-3963 (2006)

8. Y. Yang, et al., Size-dependent mechanical behavior of a-silicon carbide nanowires under in-situ transmission electron microscopy tensile tests. Mater. Res. Express 6 (2019).
9. C. Ensslen et al., Notch insensitive strength and ductility in gold nanowires. Acta Mater. 108, 317-324 (2016)

10. N. Wollschl et al., Determination of the elastic behavior of silicon nanowires within a scanning electron microscope. J. Nanomater. 2016, 36 (2016)

11. L.M. Vogl et al., Transforming layered MoS2 into functional MoO2 nanowires. Nanoscale 11(24), 11687-11695 (2019)

12. B. Peng, et al., Nanoscale testing of one-dimensional nanostructures, in Micro and Nano Mechanical Testing of Materials and Devices. 280-304 (2008).

13. G. Feng, et al., A study of the mechanical properties of nanowires using nanoindentation. J. Appl. Phys. 99(7) (2006).

14. T. Natsuki, J. Natsuki, Measurement of the elastic modulus of nanowires based on resonant frequency and boundary condition effects. Phys. E. 105, 207-211 (2019)

15. Y. Zhu, In situ nanomechanical testing of crystalline nanowires in electron microscopes. JOM 68(1), 84-93 (2016)

16. T.-H. Chang et al., On the size-dependent elasticity of pentatwinned silver nanowires. Extreme Mech. Lett. 8, 177-183 (2016)

17. A. Sedlmayr, Experimental Investigations of Deformation Pathways in Nanowires, in Schriftenreihe des Institutes für Angewandte Materialien. KIT Scientific Publishing (2012)

18. N.B. Shahjahan, Z. Hu, Effects of angular misalignment on material property characterization by nanoindentation with a cylindrical flat-tip indenter. J. Mater. Res. 32(8), 1456-1465 (2016)

19. Q. Qin et al., Measuring true Young's Modulus of a cantilevered nanowire: effect of clamping on resonance frequency. Small 8(16), 2571-2576 (2012)

20. T. Fujii, et al., Focused ion beam induced surface damage effect on the mechanical properties of silicon nanowires. J. Eng. Mater. Technol. 135(4) (2013).

21. D.-J. Zeng, Q.-S. Zheng, Resonant frequency-based method for measuring the Young's moduli of nanowires. Phys. Rev. B 76(7), 075417 (2007)

22. G. Richter et al., Ultrahigh strength single crystalline nanowhiskers grown by physical vapor deposition. Nano Lett. 9(8), 3048-3052 (2009)

23. Y. Shi et al., Determination of the natural frequency of a cantilevered $\mathrm{ZnO}$ nanowire resonantly excited by a sinusoidal electric field. Nanotechnology 18(7), 075709 (2007)

24. P. Poncharal et al., Electrostatic deflections and electromechanical resonances of carbon nanotubes. Science 283(5407), 1513-1516 (1999)

25. K.H. Liu et al., In situ probing mechanical properties of individual tungsten oxide nanowires directly grown on tungsten tips inside transmission electron microscope. Appl. Phys. Lett. 89(22), 221908 (2006)

26. O.A. Bauchau, J.I. Craig, Euler-Bernoulli beam theory, in Structural Analysis. ed. by O.A. Bauchau, J.I. Craig (Springer, Dordrecht, 2009), pp. 173-221

27. M. Li et al., Template-grown metal nanowires as resonators: performance and characterization of dissipative and elastic properties. Nano Lett. 7(11), 3281-3284 (2007)

28. B. Wu, A. Heidelberg, J.J. Boland, Mechanical properties of ultrahigh-strength gold nanowires. Nat. Mater. 4(7), 525-529 (2005)

29. Z. Fang et al., Mechanical properties of gold nanowires prepared by nanoskiving approach. Nanoscale 12(15), 8194-8199 (2020)

30. Y. Zhu, Mechanics of crystalline nanowires: an experimental perspective. Appl. Mech. Rev. 69(1) (2017).

31. L.G. Zhou, H. Huang, Are surfaces elastically softer or stiffer? Appl. Phys. Lett. 84(11), 1940-1942 (2004)

32. G. Stan et al., Surface effects on the elastic modulus of Te nanowires. Appl. Phys. Lett. 92(24), 241908 (2008) 
33. G. Pennelli, M. Totaro, A. Nannini, Correlation between surface stress and apparent Young's modulus of top-down silicon nanowires. ACS Nano 6(12), 10727-10734 (2012)

34. H. Liang, M. Upmanyu, H. Huang, Size-dependent elasticity of nanowires: nonlinear effects. Phys. Rev. B 71(24), 241403 (2005)

35. J. He, C.M. Lilley, Surface effect on the elastic behavior of static bending nanowires. Nano Lett. 8(7), 1798-1802 (2008)

36. J. He, C.M. Lilley, Surface stress effect on bending resonance of nanowires with different boundary conditions. Appl. Phys. Lett. 93(26), 263108 (2008)

37. G. Yun, H.S. Park, Surface stress effects on the bending properties of FCC metal nanowires. Phys. Rev. B 79(19), 195421 (2009)

38. G.Y. Jing et al., Surface effects on elastic properties of silver nanowires: Contact atomic-force microscopy. Phys. Rev. B 73(23), 6 (2006)
39. Y. Gan, Z. Sun, Z. Chen, Extensional vibration and size-dependent mechanical properties of single-crystal gold nanorods. J. Appl. Phys. 118(16), 164304 (2015)

40. G. Yun, H. Park, Surface stress effects on the bending properties of fcc metal nanowires. Phys. Rev. B 79, 195421 (2009)

41. L. Li, R. Lin, T.Y. Ng, Contribution of nonlocality to surface elasticity. Int. J. Eng. Sci. 152, 103311 (2020)

42. J.L. Liu et al., Large displacement of a static bending nanowire with surface effects. Phys. E. 44(10), 2050-2055 (2012)

43. S.K. Park, X.L. Gao, Bernoulli-Euler beam model based on a modified couple stress theory. J. Micromech. Microeng. 16(11), 2355-2359 (2006)

44. A. Abazari, et al., Size effects on mechanical properties of micro/ nano structures. Sensors 15 (2015). 